\title{
Molecular theory of a charged particle in a polarizable nonpolar liquid
}

\author{
F. Lado \\ Department of Physics, North Carolina State University, Raleigh, North Carolina 27695-8202
}

(Received 21 October 1996; accepted 25 November 1996)

\begin{abstract}
A generalization of liquid state theory that treats internal degrees of freedom on the same mathematical footing as orientational degrees of freedom is used to calculate the Maxwell field of a charged particle in a fluid of polarizable nonpolar molecules. The polarizable fluid is modeled by Lennard-Jones molecules with classical Drude oscillators. The electric field $E(r)$ of the charged impurity is given in terms of a screening function that yields the correct dielectric constant at large $r$ and can be computed using standard procedures for the pair correlation function of classical liquid state theory. Sample numerical results using the hypernetted-chain approximation are compared with simulation. (C) 1997 American Institute of Physics. [S0021-9606(97)50309-6]
\end{abstract}

\section{INTRODUCTION}

The strength $E(r)$ of the electric field created by a point charge $q$ immersed in a dielectric fluid is conventionally described as that of the charge in vacuum reduced by a constant factor $\epsilon$, the dielectric constant of the medium; i.e.,

$$
\mathbf{E}(\mathbf{r})=\frac{q}{\epsilon r^{2}} \hat{\mathbf{r}},
$$

where $\hat{\mathbf{r}}$ is a unit vector in the radial direction $\mathbf{r}$. At the molecular level, this relation will still hold for $r$ large, but for distances $r$ out to a few atomic diameters from the particle with point charge $q$ the Maxwell field $E(r)$ will reflect the local structure of the dielectric medium, so that $\epsilon$ is no longer constant. More generally, we may define a screening function $F(r)$ such that

$$
E(r)=\frac{q}{r^{2}}[1-F(r)] .
$$

Then for large $r$ we should expect

$$
F(r) \sim \frac{\epsilon-1}{\epsilon},
$$

while for small $r$ the finite size of the charged particle should lead to $F(r) \approx 0$ inside its first near-neighbor shell, or core region. Pollock and Alder ${ }^{1}$ and Pollock, Alder, and Pratt ${ }^{2}$ have given molecular dynamics (MD) results for the screening function $F(r)$ that display these limiting characteristics and additional structure in between. They also demonstrate that the conventional continuum theory of dielectrics using cavities of vaguely defined size is quite inadequate for nondilute media.

In this paper, we formulate a computable molecular theory of a charged particle in a polarizable nonpolar fluid. The fluid model used is that of Pratt ${ }^{3}$ and of Hoye and Stell, ${ }^{4}$ who independently studied it in mean spherical approximation. This model treats the fluctuating molecular polarization $\mathbf{p}$ as an internal molecular degree of freedom that is coupled to the external translational degrees of freedom through the usual dipole-dipole interaction. More specifically, the polarization $\mathbf{p}$ of an isolated molecule is assumed to have a Gaussian thermal distribution,

$$
f_{0}(p)=\frac{1}{\left(2 \pi \alpha_{0} / \beta\right)^{3 / 2}} \exp \left(-\frac{\beta p^{2}}{2 \alpha_{0}}\right),
$$

where $\alpha_{0}$ is the molecular polarizability and $\beta=1 / k_{B} T$, with $T$ the Kelvin temperature and $k_{B}$ Boltzmann's constant. In the dense liquid, this distribution is distorted by nearneighbor interactions into some new distribution $f(p)$ and the polarizability correspondingly becomes $\alpha>\alpha_{0}$, both quantities to be determined.

The present analysis exploits recent work ${ }^{5}$ that treats these internal degrees of freedom on the same footing as orientational degrees of freedom in molecular fluids, so that a small extension of the established formalism of molecular fluids ${ }^{6}$ serves to determine as well all the electrostatic properties of the polarizable system. The basis of this generalization is expansions in polynomials of $p$ that are constructed to be orthogonal with weight function $f(p)$. The final result derived below for the charge impurity $q$ is that its screening function $F(r)$ is given by

$$
F(r)=\frac{4 \pi \rho \alpha r^{2} g_{1}^{1}(r)}{(\alpha \beta)^{1 / 2} q},
$$

where $\rho$ is the density of the host dielectric fluid and $g_{1}^{1}(r)$ a particular coefficient in the orthogonal polynomial/spherical harmonic expansion of the charge-dipole pair distribution function $g(\mathbf{r}, \mathbf{p})$. We show further that $F(r)$ calculated in this way satisfies the asymptotic limit (3) as well as the core condition and that in general this function can be computed using the familiar equations of classical liquid state theory. ${ }^{7}$ Sample results using the hypernetted-chain approximation are given in Section V.

\section{ELECTRIC FIELD OF A CHARGE IN A POLARIZABLE FLUID}

The model consists of $N+1$ particles, all of which interact pairwise with each other through a common potential $u_{0}(r)$, which we take to be Lennard-Jones (LJ). Particle 0 carries a point charge $q$ and its location defines the origin of coordinates. It is not polarizable. The remaining $N$ particles are polarizable nonpolar molecules with molecular polarizability $\alpha_{0}$. Their instantaneous point dipoles lead to additional interactions, through dipole-dipole forces with each 
other and through charge-dipole forces with particle 0 . Thus the total potential energy of an instantaneous configuration of positions $\mathbf{r}_{j}$ and dipole moments $\mathbf{p}_{j}$ is

$$
U=U_{0}+U_{D D}+U_{C D},
$$

where

$$
\begin{aligned}
& U_{0}=\sum_{0 \leqslant i<j \leqslant N} u_{0}\left(r_{i j}\right), \\
& U_{D D}=\sum_{1 \leqslant i<j \leqslant N} u_{d d}\left(\mathbf{r}_{i j}, \mathbf{p}_{i}, \mathbf{p}_{j}\right), \\
& U_{C D}=\sum_{1 \leqslant j \leqslant N} u_{c d}\left(\mathbf{r}_{0 j}, \mathbf{p}_{j}\right),
\end{aligned}
$$

with

$$
\begin{aligned}
& u_{0}(r)=4 \epsilon_{\mathrm{LJ}}\left[\left(\frac{\sigma}{r}\right)^{12}-\left(\frac{\sigma}{r}\right)^{6}\right], \\
& u_{d d}\left(\mathbf{r}, \mathbf{p}_{1}, \mathbf{p}_{2}\right)=-\frac{1}{r^{3}}\left[3\left(\hat{\mathbf{r}} \cdot \mathbf{p}_{1}\right)\left(\hat{\mathbf{r}} \cdot \mathbf{p}_{2}\right)-\mathbf{p}_{1} \cdot \mathbf{p}_{2}\right], \\
& u_{c d}(\mathbf{r}, \mathbf{p})=-\frac{q}{r^{2}}(\hat{\mathbf{r}} \cdot \mathbf{p}) .
\end{aligned}
$$

The configurational partition function for constant $(N, V, T)$ is thus

$$
\begin{aligned}
Z & =\frac{1}{V^{N+1}} \int d \mathbf{r}_{0} \prod_{j=1}^{N}\left[d \mathbf{r}_{j} d \mathbf{p}_{j} f_{0}\left(\mathbf{p}_{j}\right)\right] \exp (-\beta U) \\
& =\frac{1}{V^{N}} \int \prod_{j=1}^{N}\left[d \mathbf{r}_{j} d \mathbf{p}_{j} f_{0}\left(\mathbf{p}_{j}\right)\right] \exp (-\beta U),
\end{aligned}
$$

where $V$ is the system volume.

Now, the electric field at point $\mathbf{r}$ due to charge $q$ at the origin and $N$ instantaneous dipoles $\mathbf{p}_{j}$ at corresponding $\mathbf{r}_{j}$ is

$$
\frac{q}{r^{2}} \hat{\mathbf{r}}+\sum_{j=1}^{N} \frac{3\left(\mathbf{p}_{j} \cdot \hat{\mathbf{R}}_{j}\right) \hat{\mathbf{R}}_{j}-\mathbf{p}_{j}}{\left|\mathbf{r}-\mathbf{r}_{j}\right|^{3}},
$$

with

$$
\hat{\mathbf{R}}_{j} \equiv \frac{\mathbf{r}-\mathbf{r}_{j}}{\left|\mathbf{r}-\mathbf{r}_{j}\right|},
$$

and so the mean field is

$$
\begin{aligned}
\mathbf{E}(\mathbf{r})= & \frac{q}{r^{2}} \hat{\mathbf{r}}+\int d \mathbf{r}^{\prime} d \mathbf{p}^{\prime} \frac{\rho f\left(p^{\prime}\right) g\left(\mathbf{r}^{\prime}, \mathbf{p}^{\prime}\right)}{\left|\mathbf{r}-\mathbf{r}^{\prime}\right|^{3}} \\
& \times\left[3 \frac{\mathbf{p}^{\prime} \cdot\left(\mathbf{r}-\mathbf{r}^{\prime}\right)}{\left|\mathbf{r}-\mathbf{r}^{\prime}\right|^{2}}\left(\mathbf{r}-\mathbf{r}^{\prime}\right)-\mathbf{p}^{\prime}\right],
\end{aligned}
$$

where

$$
\rho f(p) g(\mathbf{r}, \mathbf{p})=\left\langle\sum_{j=1}^{N} \delta\left(\mathbf{r}-\mathbf{r}_{j}\right) \delta\left(\mathbf{p}-\mathbf{p}_{j}\right)\right\rangle
$$

defines the pair distribution function $g(\mathbf{r}, \mathbf{p})$ of particle 0 with the polarizable molecules of instantaneous polarization p. Here the angular brackets denote a canonical ensemble average with particle 0 fixed at the origin.

We seek to evaluate the integrals of Eq. (15) without yet knowing $g(\mathbf{r}, \mathbf{p})$; i.e., to determine what is minimally needed from this function to find the Maxwell field. To this end, it is useful to explicitly break out its dependence on the direction and magnitude of $\mathbf{p}$. With the $z$ axis aligned along $\mathbf{r}$, we first write

$$
\begin{aligned}
g(\mathbf{r}, \mathbf{p}) & =g(r, p, \cos \theta) \\
& =\sum_{l} g_{l}(r, p)(2 l+1)^{1 / 2} P_{l}(\cos \theta),
\end{aligned}
$$

where $\theta$ is the polar angle between $\mathbf{r}$ and $\mathbf{p}$ and $P_{l}(x)$ the Legendre polynomial of order $l$. The orthogonality of these polynomials,

$$
\frac{1}{2}(2 l+1) \int_{-1}^{1} d x P_{l}(x) P_{l^{\prime}}(x)=\delta_{l l^{\prime}},
$$

allows the easy inversion of the expansion. Similarly, we then expand

$$
g_{l}(r, p)=\sum_{n} g_{l}^{n}(r) Q_{n l}(p),
$$

where the $Q_{n l}(p)$ are polynomials of $p$ constructed to be orthogonal with weight function $f(p)$,

$$
4 \pi \int_{0}^{\infty} d p p^{2} f(p) Q_{n l}(p) Q_{n^{\prime} l}(p)=\delta_{n n^{\prime}},
$$

so that (19) also has an easy inversion. Consider now the integral over $\mathbf{p}^{\prime}$ in Eq. (15),

$$
\mathbf{w}\left(\mathbf{r}^{\prime}\right) \equiv \int d \mathbf{p}^{\prime} f\left(p^{\prime}\right) g\left(\mathbf{r}^{\prime}, \mathbf{p}^{\prime}\right) \mathbf{p}^{\prime} .
$$

Since this is a vector quantity that depends only on $\mathbf{r}^{\prime}$, it must be of the form $\mathbf{w}\left(\mathbf{r}^{\prime}\right)=w\left(r^{\prime}\right) \hat{\mathbf{r}}^{\prime}$, where then

$$
\begin{aligned}
w\left(r^{\prime}\right)= & \int d \mathbf{p}^{\prime} f\left(p^{\prime}\right) g\left(\mathbf{r}^{\prime}, \mathbf{p}^{\prime}\right) \hat{\mathbf{r}}^{\prime} \cdot \mathbf{p}^{\prime} \\
= & \sum_{n, l} g_{l}^{n}\left(r^{\prime}\right) 4 \pi \int_{0}^{\infty} d p^{\prime} p^{\prime 2} f\left(p^{\prime}\right) p^{\prime} Q_{n l}\left(p^{\prime}\right) \\
& \times \frac{1}{2}(2 l+1)^{1 / 2} \int_{-1}^{1} d x x P_{l}(x) \\
= & \left(\frac{\alpha}{\beta}\right)^{1 / 2} g_{1}^{1}\left(r^{\prime}\right) .
\end{aligned}
$$

In addition to the expressed orthogonalities, we have used here the specific form (see the next section) $Q_{11}(p)$ $=(\beta / 3 \alpha)^{1 / 2} p$.

Returning to Eq. (15), we now have 


$$
\begin{aligned}
\mathbf{E}(\mathbf{r})= & \frac{q}{r^{2}} \hat{\mathbf{r}}+\left(\frac{\alpha}{\beta}\right)^{1 / 2} \rho \int d \mathbf{r}^{\prime} \frac{g_{1}^{1}\left(r^{\prime}\right)}{\left|\mathbf{r}-\mathbf{r}^{\prime}\right|^{3}} \\
& \times\left[3 \frac{\hat{\mathbf{r}}^{\prime} \cdot\left(\mathbf{r}-\mathbf{r}^{\prime}\right)}{\left|\mathbf{r}-\mathbf{r}^{\prime}\right|^{2}}\left(\mathbf{r}-\mathbf{r}^{\prime}\right)-\hat{\mathbf{r}}^{\prime}\right]
\end{aligned}
$$

Again by symmetry, the second term must also be in the direction $\hat{\mathbf{r}}$, so that the magnitude of the Maxwell field is

$$
\begin{aligned}
E(r)= & \frac{q}{r^{2}}-\left(\frac{\alpha}{\beta}\right)^{1 / 2} \rho \int d \mathbf{r}^{\prime} \frac{g_{1}^{1}\left(r^{\prime}\right)}{\left|\mathbf{r}-\mathbf{r}^{\prime}\right|^{3}} \\
& \times\left\{3 \frac{\left[\hat{\mathbf{r}} \cdot\left(\mathbf{r}-\mathbf{r}^{\prime}\right)\right]\left[\hat{\mathbf{r}}^{\prime} \cdot\left(\mathbf{r}^{\prime}-\mathbf{r}\right)\right]}{\left|\mathbf{r}-\mathbf{r}^{\prime}\right|^{2}}+\hat{\mathbf{r}} \cdot \hat{\mathbf{r}}^{\prime}\right\} \\
= & \frac{q}{r^{2}}-\left(\frac{\alpha}{\beta}\right)^{1 / 2} 2 \pi \rho \int_{0}^{\infty} d r^{\prime} r^{\prime 2} g_{1}^{1}\left(r^{\prime}\right) \Delta\left(r, r^{\prime}\right),
\end{aligned}
$$

where

$$
\begin{aligned}
\Delta\left(r, r^{\prime}\right) \equiv & \int_{-1}^{1} d x\left[3 \frac{\left(r-r^{\prime} x\right)\left(r^{\prime}-r x\right)}{\left(r^{2}+r^{\prime 2}-2 r r^{\prime} x\right)^{5 / 2}}\right. \\
& \left.+\frac{x}{\left(r^{2}+r^{\prime 2}-2 r r^{\prime} x\right)^{3 / 2}}\right] .
\end{aligned}
$$

It is then easy to show that

$$
\begin{aligned}
\Delta\left(r, r^{\prime}\right) & =\frac{d^{2}}{d r d r^{\prime}} \int_{-1}^{1} d x \frac{1}{\left(r^{2}+r^{\prime 2}-2 r r^{\prime} x\right)^{1 / 2}} \\
& =\frac{d^{2}}{d r d r^{\prime}}\left[\frac{2}{r} H\left(r-r^{\prime}\right)+\frac{2}{r^{\prime}} H\left(r^{\prime}-r\right)\right] \\
& =\frac{2}{r^{2}} \delta\left(r-r^{\prime}\right),
\end{aligned}
$$

where $H(x)$ is the Heaviside unit function and $\delta(x)=d H(x) / d x$ the Dirac delta function. The final result is thus

$$
E(r)=\frac{q}{r^{2}}-\left(\frac{\alpha}{\beta}\right)^{1 / 2} 4 \pi \rho g_{1}^{1}(r)=\frac{q}{r^{2}}[1-F(r)],
$$

so that the screening function $F(r)$ is

$$
F(r)=\frac{4 \pi \rho \alpha r^{2} g_{1}^{1}(r)}{(\alpha \beta)^{1 / 2} q},
$$

as noted earlier. The asymptotic limit of $F(r)$ is found in Section IV after we examine the calculation of $g(\mathbf{r}, \mathbf{p})$ in the next section.

\section{CALCULATION OF $g(r, p)$}

The system composed of charge $q$ immersed in a fluid of $N$ polarizable molecules constitutes a two-component mixture with one component at infinite dilution. We can thus adapt the usual equations for liquid state mixtures ${ }^{7}$ to calculate the charge-dipole and dipole-dipole correlation functions, $h\left(\mathbf{r}_{01}, \mathbf{p}_{1}\right)$ and $h\left(\mathbf{r}_{12}, \mathbf{p}_{1}, \mathbf{p}_{2}\right)$, respectively, where $h=g-1$. These are the Ornstein-Zernike (OZ) equations,

$$
\begin{aligned}
h\left(\mathbf{r}_{01}, \mathbf{p}_{1}\right)= & c\left(\mathbf{r}_{01}, \mathbf{p}_{1}\right) \\
& +\rho \int d \mathbf{r}_{2} d \mathbf{p}_{2} f\left(p_{2}\right) h\left(\mathbf{r}_{02}, \mathbf{p}_{2}\right) c\left(\mathbf{r}_{21}, \mathbf{p}_{2}, \mathbf{p}_{1}\right),
\end{aligned}
$$

$$
\begin{aligned}
h\left(\mathbf{r}_{12}, \mathbf{p}_{1}, \mathbf{p}_{2}\right)= & c\left(\mathbf{r}_{12}, \mathbf{p}_{1}, \mathbf{p}_{2}\right) \\
& +\rho \int d \mathbf{r}_{3} d \mathbf{p}_{3} f\left(p_{3}\right) h\left(\mathbf{r}_{13}, \mathbf{p}_{1}, \mathbf{p}_{3}\right) \\
& \times c\left(\mathbf{r}_{32}, \mathbf{p}_{3}, \mathbf{p}_{2}\right),
\end{aligned}
$$

and the closure equations

$$
\begin{aligned}
c\left(\mathbf{r}, \mathbf{p}_{1}\right)= & h\left(\mathbf{r}, \mathbf{p}_{1}\right)-\ln \left[1+h\left(\mathbf{r}, \mathbf{p}_{1}\right)\right]-\beta u_{0}(r) \\
- & \beta u_{c d}\left(\mathbf{r}, \mathbf{p}_{1}\right)+B\left(\mathbf{r}, \mathbf{p}_{1}\right), \\
c\left(\mathbf{r}, \mathbf{p}_{1}, \mathbf{p}_{2}\right)= & h\left(\mathbf{r}, \mathbf{p}_{1}, \mathbf{p}_{2}\right)-\ln \left[1+h\left(\mathbf{r}, \mathbf{p}_{1}, \mathbf{p}_{2}\right)\right]-\beta u_{0}(r) \\
& -\beta u_{d d}\left(\mathbf{r}, \mathbf{p}_{1}, \mathbf{p}_{2}\right)+B\left(\mathbf{r}, \mathbf{p}_{1}, \mathbf{p}_{2}\right) .
\end{aligned}
$$

Here, $c\left(\mathbf{r}_{01}, \mathbf{p}_{1}\right)$ and $c\left(\mathbf{r}_{12}, \mathbf{p}_{1}, \mathbf{p}_{2}\right)$ are the corresponding direct correlation functions of the mixture. As usual, the closure relations must be supplemented with some approximation for the so-called bridge functions, $B\left(\mathbf{r}_{01}, \mathbf{p}_{1}\right)$ and $B\left(\mathbf{r}_{12}, \mathbf{p}_{1}, \mathbf{p}_{2}\right)$.

The solution of the dipole-dipole equations has already been described in a recent publication. ${ }^{5}$ Here we need be concerned only with the calculation for $h\left(\mathbf{r}_{01}, \mathbf{p}_{1}\right)$. As in the earlier work, the first step in the solution is the expansion of all functions of $\mathbf{p}$ in polynomials $Q_{n l}(p)$ and spherical harmonics $Y_{l m}(\omega)$; e.g.,

$$
\begin{aligned}
h(\mathbf{r}, \mathbf{p}) & =(4 \pi)^{1 / 2} \sum_{n, l} h_{l}^{n}(r) Q_{n l}(p) Y_{l 0}(\omega) \\
& =\sum_{n, l} h_{l}^{n}(r) Q_{n l}(p) \mathscr{P}_{l}(\cos \theta),
\end{aligned}
$$

as in Section II, with $\mathscr{P}_{l}(x)=(2 l+1)^{1 / 2} P_{l}(x)$, and

$$
\begin{aligned}
h\left(\mathbf{r}, \mathbf{p}_{1}, \mathbf{p}_{2}\right)= & 4 \pi \sum_{n_{1}, n_{2}, l_{1}, l_{2}, m} h_{l_{1} l_{2} m}^{n_{1} n_{2}}(r) Q_{n_{1} l_{1}}\left(p_{1}\right) Q_{n_{2} l_{2}}\left(p_{2}\right) \\
& \times Y_{l_{1} m}\left(\omega_{1}\right) Y_{l_{2} \bar{m}}\left(\omega_{2}\right) .
\end{aligned}
$$

Equations (29) and (31) are then rewritten in terms of the indirect correlation function $\gamma=h-c$ and the $\mathrm{OZ}$ equation is deconvoluted using Fourier transforms. After introducing the corresponding expansions of the transforms, we get for the $\mathrm{OZ}$ equation,

$$
\widetilde{\gamma}_{l}^{n}(k)=\rho \sum_{n^{\prime}, l^{\prime}}\left[\widetilde{\gamma}_{l^{\prime}}^{n^{\prime}}(k)+\widetilde{c}_{l^{\prime}}^{n^{\prime}}(k)\right] \widetilde{c}_{l^{\prime} l 0}^{n^{\prime} n}(k),
$$

which can be further reduced to read, in matrix notation,

$$
\widetilde{\Gamma}(k)=\rho \widetilde{H}_{0}(k) \widetilde{C}(k) .
$$

Here, $\widetilde{\Gamma}(k)$ and $\widetilde{C}(k)$ are column matrices with elements $\widetilde{\gamma}_{l}^{n}(k)$ and $\widetilde{c}_{l}^{n}(k)$, respectively, while $\widetilde{H}_{0}(k)$ is a square matrix with elements ${\widetilde{h_{l l}^{\prime}}}_{l{ }^{\prime} 0}{ }^{\prime}(k) .^{5}$ The closure equation meanwhile becomes 
$c(r, p, x)=g(r, p, x)-1-\gamma(r, p, x)$,

with

$$
\begin{aligned}
g(r, p, x)= & \exp \left[-\beta u_{0}(r)-\beta u_{c d}(r, p, x)+\gamma(r, p, x)\right. \\
& +B(r, p, x)] .
\end{aligned}
$$

The solution of the nonlinear equations for the $\gamma_{l}^{n}(r)$ is iterative. One iteration consists of the following four steps:

(i) Closure. Numerically evaluate

$$
g_{l}^{n}(r)=\int d \mathbf{p} f(p) g(r, p, \cos \theta) Q_{n l}(p) \mathscr{P}_{l}(\cos \theta)
$$

using Gaussian quadratures, with $g(r, p, \cos \theta)$ given by Eq. (38), and form

$$
c_{l}^{n}(r)=g_{l}^{n}(r)-\delta_{n 0} \delta_{l 0}-\gamma_{l}^{n}(r) .
$$

(ii) Transforms. Compute the Fourier transforms

$$
\widetilde{c}_{l}^{n}(k)=4 \pi i^{l} \int_{0}^{\infty} d r r^{2} c_{l}^{n}(r) j_{l}(k r),
$$

where $j_{l}(x)$ is the spherical Bessel function of order $l$. from

(iii) OZ equation. Obtain the matrix elements $\widetilde{\gamma}_{l}^{n}(k)$

$$
\widetilde{\Gamma}(k)=\rho \widetilde{H}_{0}(k) \widetilde{C}(k) .
$$

(iv) Inverse transforms. Compute the inverse Fourier transforms

$$
\gamma_{l}^{n}(r)=\frac{1}{2 \pi^{2} i^{l}} \int_{0}^{\infty} d k k^{2} \widetilde{\gamma}_{l}^{n}(k) j_{l}(k r) .
$$

These new coefficients $\gamma_{l}^{n}(r)$ are then used to reconstruct $\gamma(r, p, x)$ in step $(i)$ and initiate a new cycle. [Most approximations for $B(r, p, x)$ are also expressed as functions of $\gamma(r, p, x)$.] Iterations are continued until a self-consistent set of coefficients $\gamma_{l}^{n}(r)$ is obtained.

Other details of the calculation are similar to those of the earlier work. ${ }^{5}$ We add here for completeness that the distribution $f(p)$ is found to remain essentially Gaussian for the state studied in Section V, as earlier in Ref. 5, so that the polynomials $Q_{n l}(p)$ are $^{8}$

$$
\begin{aligned}
Q_{n l}(p)= & {\left[\frac{\Gamma\left(\frac{1}{2}(n-l)+1\right) \Gamma\left(\frac{3}{2}\right)}{\Gamma\left(\frac{1}{2}(n+l)+\frac{3}{2}\right)}\right]^{1 / 2} } \\
& \times\left(\frac{\beta p^{2}}{2 \alpha}\right)^{l / 2} L_{\frac{1}{2}(n-l)}^{l+1 / 2}\left(\frac{\beta p^{2}}{2 \alpha}\right),
\end{aligned}
$$

where $L_{n}^{b}(t)$ is an associated Laguerre polynomial ${ }^{9,10}$ and $\Gamma(z)$ is the gamma function. The indices range over $n=0,1,2,3, \ldots$ and $l=n, n-2, n-4, \ldots, 1$ or 0 .

\section{ASYMPTOTIC LIMITS AND FREE ENERGY CHANGES}

The charge-dipole potential $\beta u_{c d}(\mathbf{r}, \mathbf{p})$ has the single expansion coefficient

$$
\beta u_{1}^{1}(r)=-\frac{(\alpha \beta)^{1 / 2} q}{r^{2}},
$$

whose Fourier transform is

$$
\beta \tilde{u}_{1}^{1}(k)=4 \pi i \int_{0}^{\infty} d r r^{2} \beta u_{1}^{1}(r) j_{1}(k r)=-\frac{4 \pi i(\alpha \beta)^{1 / 2} q}{k} \text {. }
$$

Now, for small $k$, the dominant term in Eq. (42) for $\widetilde{\gamma}_{1}^{1}(k)$ is

$$
\widetilde{\gamma}_{1}^{1}(k) \approx \rho \widetilde{h}_{110}^{11}(0) \widetilde{c}_{1}^{1}(k),
$$

so that, in the same limit,

$$
\begin{aligned}
\widetilde{h}_{1}^{1}(k) & \approx\left[1+\rho \widetilde{h}_{110}^{11}(0)\right] \widetilde{c}_{1}^{1}(k) \\
& \approx-\left[1+\rho \widetilde{h}_{110}^{11}(0)\right] \beta \widetilde{u}_{1}^{1}(k),
\end{aligned}
$$

since inversely, for large $r$,

$$
c_{1}^{1}(r) \sim-\beta u_{1}^{1}(r) .
$$

Here we recall from Ref. 5 that

$$
1+\rho \widetilde{h}_{110}^{11}(0)=\frac{\epsilon-1}{4 \pi \rho \alpha \epsilon},
$$

and so we find finally that, for small k,

$$
\tilde{h}_{1}^{1}(k) \approx \frac{\epsilon-1}{\epsilon} \frac{i(\alpha \beta)^{1 / 2} q}{\rho \alpha k} .
$$

Comparing this with the transform $\beta \tilde{u}_{1}^{1}(k)$ of the potential $\beta u_{1}^{1}(r)$ we see that, for large $r$, we must have

$$
h_{1}^{1}(r)=g_{1}^{1}(r) \sim \frac{\epsilon-1}{\epsilon} \frac{(\alpha \beta)^{1 / 2} q}{4 \pi \rho \alpha r^{2}},
$$

and hence that

$$
F(r)=\frac{4 \pi \rho \alpha r^{2} g_{1}^{1}(r)}{(\alpha \beta)^{1 / 2} q} \sim \frac{\epsilon-1}{\epsilon},
$$

as required.

Pollock and Alder ${ }^{1}$ have used their simulation results for the screening function to calculate the free energy of the ion in solution. Here we follow their use of the Onsager charging process to rederive the same result in the new formalism. From the partition function of Eq. (13), we get the free en$\operatorname{ergy} A$ of the system as $\beta A=-\ln Z$, and so

$$
\begin{aligned}
\frac{\partial \beta A}{\partial q} & =-\frac{1}{Z V^{N}} \int \prod_{j=1}^{N}\left[d \mathbf{r}_{j} d \mathbf{p}_{j} f_{0}\left(\mathbf{p}_{j}\right)\right] e^{-\beta U} \beta \sum_{j=1}^{N} \frac{\hat{\mathbf{r}}_{0 j} \cdot \mathbf{p}_{j}}{r_{0 j}^{2}} \\
& =-N\left(\beta \frac{\hat{\mathbf{r}}_{01} \cdot \mathbf{p}_{1}}{r_{01}^{2}}\right) \\
& =-N \rho \int d \mathbf{r} d \mathbf{p} f(p) g(\mathbf{r}, \mathbf{p}) \beta \frac{\hat{\mathbf{r}} \cdot \mathbf{p}}{r^{2}}
\end{aligned}
$$

After inserting the expansion (33) and using the orthogonality relations, we find

$$
\frac{\partial(\beta A / N)}{\partial q}=-(\alpha \beta)^{1 / 2} 4 \pi \rho \int_{0}^{\infty} d r g_{1}^{1}(r) .
$$




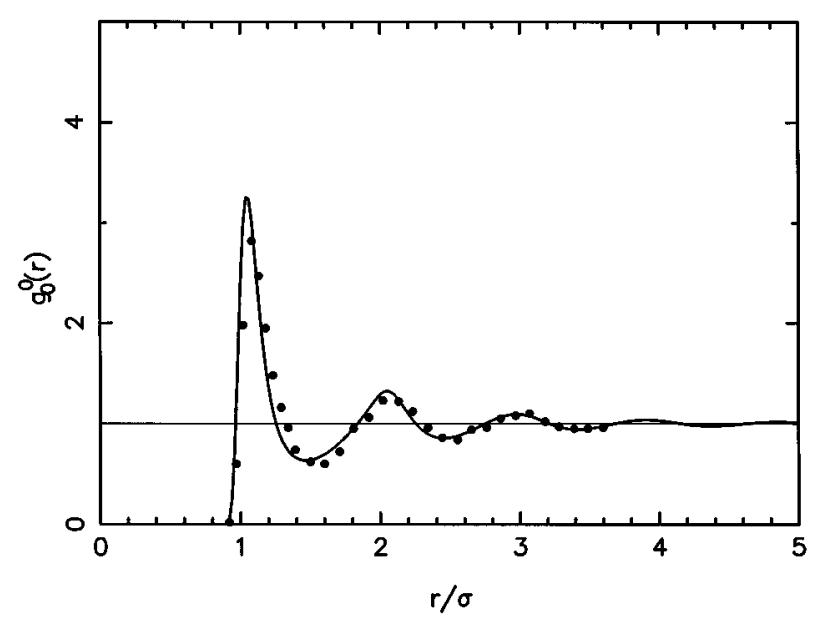

FIG. 1. Radial distribution function $g_{0}^{0}(r)$ of polarizable LJ molecules about a charged LJ particle of reduced charge $q /\left(\epsilon_{\mathrm{LJ}} \sigma\right)^{1 / 2}=0.01$ : solid line HNC; points - MD (Ref. 2). The state of the polarizable liquid is $\rho \sigma^{3}=0.844, k_{B} T / \epsilon_{\mathrm{LJ}}=0.85, \alpha_{0} / \sigma^{3}=0.10$.

In terms of the screening function $F(r)$, this is equivalently written as

$$
\frac{\partial(\beta A / N)}{\partial q}=-\int_{0}^{\infty} d r F(r) \frac{\beta q}{r^{2}} .
$$

Comparison with Pollock and Alder's result ${ }^{1,2}$ shows that their screening function $S(r)$ is related to $F(r)$ according to

$$
F(r)=4 \pi \rho \alpha_{0} g_{0}^{0}(r) S(r),
$$

where $g_{0}^{0}(r)$ is the radial distribution function of polarizable molecules about the charge impurity.

\section{SAMPLE CALCULATIONS}

The only available simulation data for a model of a charged particle in a polarizable nonpolar fluid appear to be those of Pollock and Alder ${ }^{1,2}$ for a liquid state of LennardJones molecules near the triple point: $\rho \sigma^{3}=0.844$, $k_{B} T / \epsilon_{\mathrm{LJ}}=0.85, \alpha_{0} / \sigma^{3}=0.10$. Thus all calculations in this section are for this same state. (The computed values ${ }^{5}$ of $\alpha$ and $\epsilon$ for this state are $\alpha / \sigma^{3}=0.1115$ and $\epsilon=2.699$.)

Exploratory calculations with several closures lead to the conclusion that in the present case the hypernetted-chain (HNC) approximation, $B(\mathbf{r}, \mathbf{p}) \approx 0$, is the best available for the least computational effort. The HNC results reported below use nine coefficients of $\gamma(\mathbf{r}, \mathbf{p})$ (see below, however, for an exception); arranged as a row matrix, they are

$$
\left[\gamma_{0}^{0}(r) \gamma_{0}^{2}(r) \gamma_{0}^{4}(r) \gamma_{1}^{1}(r) \gamma_{1}^{3}(r) \gamma_{2}^{2}(r) \gamma_{2}^{4}(r) \gamma_{3}^{3}(r) \gamma_{4}^{4}(r)\right]
$$

and match the largest set of dipole-dipole coefficients used here for $\widetilde{H}_{0}(k)$ in Eq. (42) and earlier in Ref. 5. Integrals in $r$ and $k$ space are evaluated using $N=2048$ points and intervals $\Delta r=0.02 \sigma$ and $\Delta k=\pi / N \Delta r$, while the Gaussian quadratures of step (i), Eq. (39), are carried out with 12 points each.

Figures 1 and 2 show the radial distribution function $g_{0}^{0}(r)$ for two different charges, $q /\left(\epsilon_{\mathrm{LJ}} \sigma\right)^{1 / 2}=0.01$ and 10 .

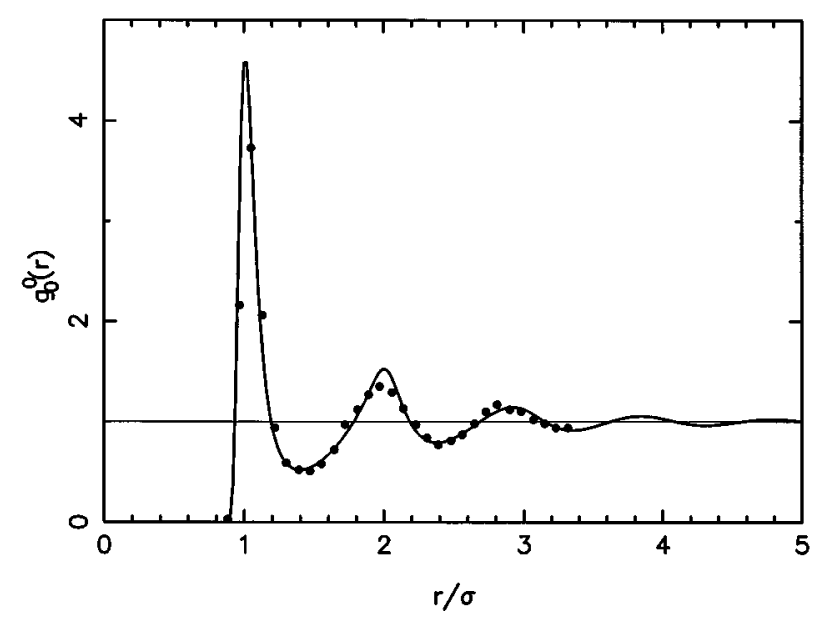

FIG. 2. Radial distribution function $g_{0}^{0}(r)$ of polarizable LJ molecules about a charged LJ particle of reduced charge $q /\left(\epsilon_{\mathrm{LJ}} \sigma\right)^{1 / 2}=10$ : solid line - HNC; points - MD (Ref. 2). The polarizable liquid state is the same as in Fig. 1.

The first is essentially the neutral atom limit while the second corresponds to about half an electronic charge, $q \approx 0.5 e$, if argon values are used for the LJ parameters: $\sigma=3.4 \AA$ and $\epsilon_{\mathrm{LJ}}=120 k_{B}$, so $e /\left(\epsilon_{\mathrm{LJ}} \sigma\right)^{1 / 2}=20.2$. The figures are drawn to the same scale so that the increase in the packing of polarizable molecules about the larger charge is readily apparent. Comparison of the HNC results with the simulation data shows reasonably good agreement.

The corresponding screening functions $F(r)$ [essentially rescaled versions of the coefficients $\left.g_{1}^{1}(r)\right]$ are shown in Figs. 3 and 4, again to the same scale. The comments made above about the two $g_{0}^{0}(r)$ curves apply here as well. This reflects the fact that the screening function $S(r)$ [or $\left.r^{2} g_{1}^{1}(r) / g_{0}^{0}(r)\right]$ defined by Pollock and Alder is comparatively flat, so that $F(r)$ in Eq. (57) strongly echoes the structure of $g_{0}^{0}(r)$. [In fact, a simple approximation that captures the structural essence is $F(r) \approx[(\epsilon-1) / \epsilon] g_{0}^{0}(r)$. $]$ Compari-

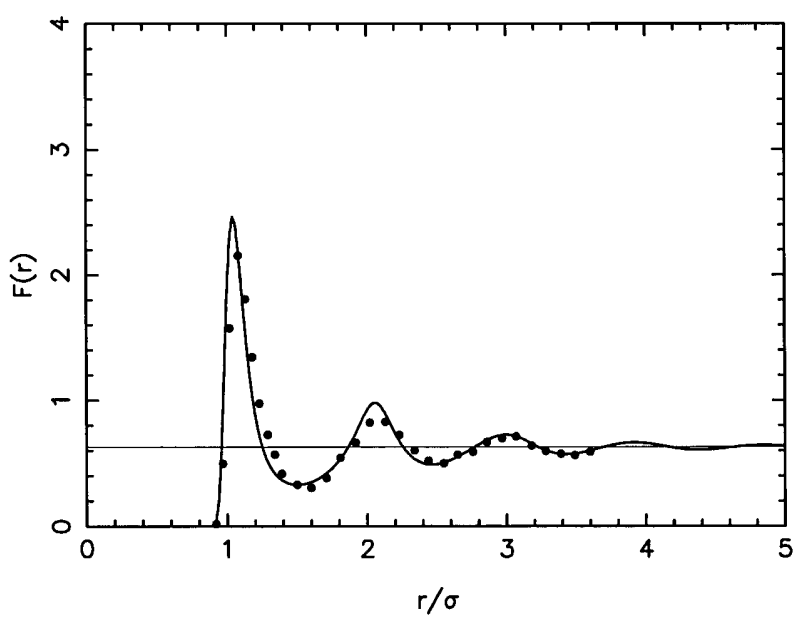

FIG. 3. Screening function $F(r)$ for a reduced charge $q /\left(\epsilon_{\mathrm{LJ}} \sigma\right)^{1 / 2}=0.01$ in a liquid of polarizable molecules in the same state as in Fig. 1: solid line HNC; points - MD (Ref. 2). The light horizontal line is the asymptotic limit $(\epsilon-1) / \epsilon=0.63$. 


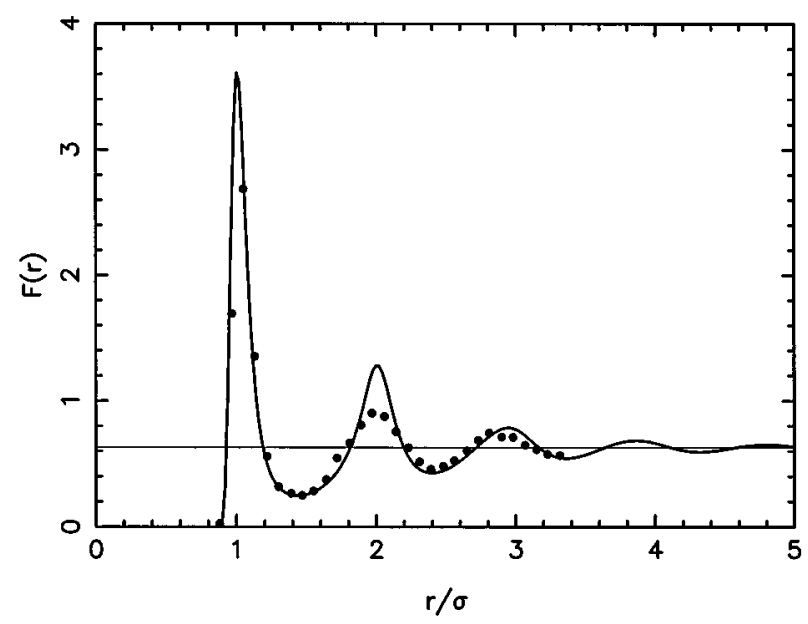

FIG. 4. Screening function $F(r)$ for a reduced charge $q /\left(\epsilon_{\mathrm{LJ}} \sigma\right)^{1 / 2}=10$ in a liquid of polarizable molecules in the same state as in Fig. 1: solid line HNC; points - MD (Ref. 2). The light horizontal line is the asymptotic limit $(\epsilon-1) / \epsilon=0.63$.

son with simulation results shows that the HNC approximation is noticeably overestimating the second near-neighbor peak. In both cases, the asymptotic limit of $(\epsilon-1) / \epsilon$ is reached in a few diameters $\sigma$.

Figures 5 and 6 illustrate functional properties that were used in the arguments of Section IV. In Fig. 5 we show the coefficient $c_{1}^{1}(r)$ of the direct correlation function $c(\mathbf{r}, \mathbf{p})$ for charge $q /\left(\epsilon_{\mathrm{LJ}} \sigma\right)^{1 / 2}=10$; it is seen to blend smoothly into its asymptotic limit of $-\beta u_{1}^{1}(r)$ in about three diameters $\sigma$. (It is also notable for a sharp spike at $r=\sigma$ that grows with the charge $q$.) Figure 6 details the behavior of the coefficients $\rho \widetilde{h}_{0}^{0}(k)$ and $i \rho \widetilde{h}_{1}^{1}(k)$ of the structure function $\rho \widetilde{h}(\mathbf{k}, \mathbf{p})$, also for the charge $q /\left(\epsilon_{\mathrm{LJ}} \sigma\right)^{1 / 2}=10$, contrasted with that of $\rho \widetilde{h}_{000}^{00}(k)$ and $\rho \widetilde{h}_{110}^{11}(k)$ of the pure dipole-dipole system. The latter are seen to be nearly constant for small $k$, a fact used in Sec. IV in finding the small- $k$ form of $\rho \widetilde{h}_{1}^{1}(k)$.

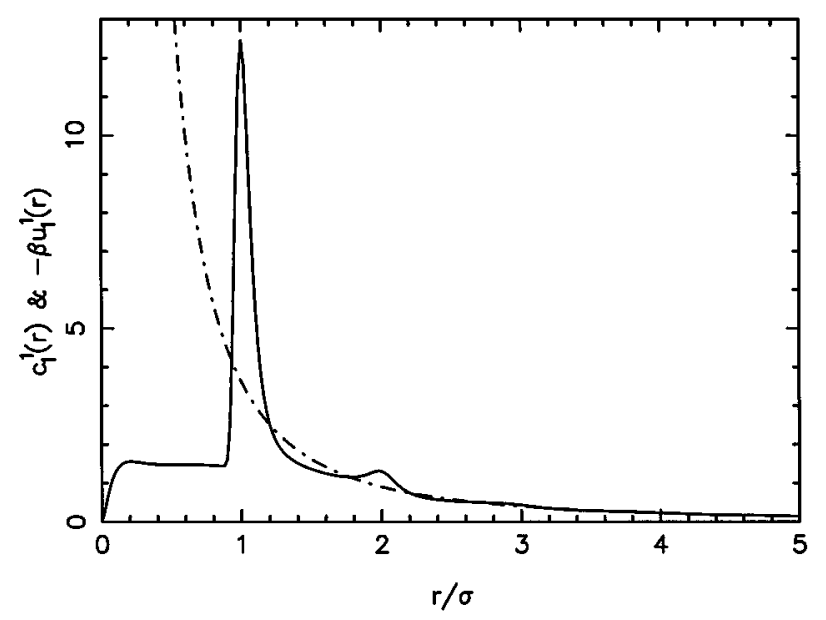

FIG. 5. HNC solution for the coefficient $c_{1}^{1}(r)$ (solid line) of the direct correlation function $c(\mathbf{r}, \mathbf{p})$ for a reduced charge $q /\left(\epsilon_{\mathrm{LJ}} \sigma\right)^{1 / 2}=10$ in a polarizable liquid in the same state as in Fig. 1, compared with its asymptotic limit, $-\beta u_{1}^{1}(r)$ (dash-dot line).

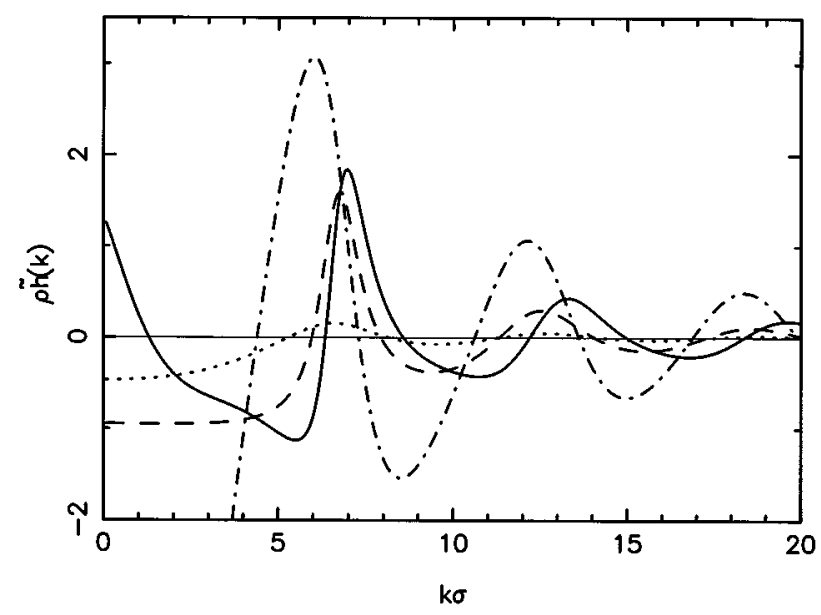

FIG. 6. HNC solution for the coefficients $\rho \widetilde{h}_{0}^{0}(k)$ (solid line) and $\rho \widetilde{h}_{1}^{1}(k)$ (dash-dot line) of the transform $\rho \widetilde{h}(\mathbf{k}, \mathbf{p})$ for a reduced charge $q /\left(\epsilon_{\mathrm{LJ}} \sigma\right)^{1 / 2}=10$ in a polarizable liquid in the same state as in Fig. 1, compared with $\rho \widetilde{h}_{000}^{00}(k)$ (dashed line) and $\rho \widetilde{h}_{110}^{11}(k)$ (dotted line) for the dipoledipole correlations, from Ref. 5.

The energy $E_{q}$ of the charge in solution is given by

$$
\begin{aligned}
\frac{\beta E_{q}}{N} & =4 \pi \rho \int_{0}^{\infty} d r r^{2}\left[g_{0}^{0}(r) \beta u_{0}^{0}(r)+g_{1}^{1}(r) \beta u_{1}^{1}(r)\right] \\
& =\frac{\beta \bar{U}_{0 q}}{N}+q \frac{\partial(\beta A / N)}{\partial q} .
\end{aligned}
$$

Here the second term involves the long-ranged coefficient $g_{1}^{1}(r)$. If $R_{c}$ is the cutoff distance in the numerical calculation, then this term is evaluated as

$$
\frac{\partial(\beta A / N)}{\partial q}=-(\alpha \beta)^{1 / 2} 4 \pi \rho \int_{0}^{R_{c}} d r g_{1}^{1}(r)-\frac{\epsilon-1}{\epsilon} \frac{\beta q}{R_{c}},
$$

where we have used the asymptotic form (52). Table I lists the computed $\mathrm{HNC}$ values for the $\mathrm{LJ}$ contribution to the

TABLE I. Lennard-Jones energy $\bar{U}_{0 q}$ and changes in free energy $A$ for a charged Lennard-Jones particle in a liquid of polarizable Lennard-Jones molecules near the triple point: $\rho \sigma^{3}=0.844, \quad k_{B} T / \epsilon_{\mathrm{LJ}}=0.85$, $\alpha_{0} / \sigma^{3}=0.10$. Comparison of computed HNC results with molecular dynamics simulation (Ref. 1). For this thermodynamic state, the HNC closure does not yield a solution for charge $q=0.75 e$ and larger, where $e$ is the electronic charge.

\begin{tabular}{ccccccc}
\hline \hline & $-\beta \bar{U}_{0 q} / N$ & \multicolumn{2}{c}{$-e \partial(\beta A / N) / \partial q$} & & \multicolumn{2}{c}{$-\beta \Delta A / N$} \\
\cline { 3 - 4 } \cline { 6 - 7 }$q / e$ & $\mathrm{HNC}$ & $\mathrm{HNC}$ & $\mathrm{MD}$ & & $\mathrm{HNC}$ & $\mathrm{MD}$ \\
\hline 0 & 12.91 & 0 & 0 & & 0 \\
0.10 & 12.85 & 41.5 & & & 2.1 & \\
0.20 & 12.63 & 84.2 & & & 8.3 & \\
0.25 & 12.44 & 106.5 & 108 & & 13.1 & 13 \\
0.30 & 12.17 & 129.7 & & & 18.9 & \\
0.40 & 11.33 & 179.9 & & & 34.4 & \\
0.50 & 9.81 & 237.7 & 234 & & 55.3 & 55 \\
0.60 & 6.88 & 310.6 & & 82.5 & \\
0.70 & 2.50 & 395.6 & & & 117.3 & \\
0.75 & & & 380 & & 130 \\
\hline \hline
\end{tabular}


energy of the charge $q$ in solution, $\beta \bar{U}_{0 q} / N$, and the rate of change of the free energy with charge. The latter quantities, along with others obtained for midinterval points $q /\left(\epsilon_{\mathrm{LJ}} \sigma\right)^{1 / 2}=0.05,0.15, \ldots$, are then fit to a polynomial of the form

$$
e \frac{\partial(\beta A / N)}{\partial q}=-\left[a_{0}+a_{1}\left(\frac{q}{e}\right)+a_{2}\left(\frac{q}{e}\right)^{2}\right] \frac{q}{e},
$$

using least squares, so that the total free energy change is obtained as

$$
\frac{\beta \Delta A}{N}=-\left[\frac{1}{2} a_{0}\left(\frac{q}{e}\right)^{2}+\frac{1}{3} a_{1}\left(\frac{q}{e}\right)^{3}+\frac{1}{4} a_{2}\left(\frac{q}{e}\right)^{4}\right] .
$$

The computed coefficients are $a_{0}=418.0, a_{1}=-70.9$, and $a_{2}=382.8$. Comparison of the free energy results with the simulation data of Pollock and Alder ${ }^{1}$ shows good agreement.

A disappointing feature of Table $\mathrm{I}$ is that it is prematurely truncated. While Pollock and Alder ${ }^{1}$ go on to give similar results for charges $q$ up to $2 e$, the HNC approximation does not yield converged solutions for this thermodynamic state at $q=0.75 e$ and larger. (Such solutions can be obtained at higher temperatures, however.) In fact, the results for $q / e=0.65$ and 0.70 could be obtained only by omitting the three coefficients $\gamma_{l}^{n}(r)$ with an index 4 from the previously given set. (The corresponding data points were not used in the least squares fit.) Whether this convergence problem is inherent in the HNC approximation or can be remedied by a modification of the numerical algorithm used is not yet known.

${ }^{1}$ E. L. Pollock and B. J. Alder, Phys. Rev. Lett. 41, 903 (1978).

${ }^{2}$ E. L. Pollock, B. J. Alder, and L. R. Pratt, Proc. Natl. Acad. Sci. USA 77, 49 (1980).

${ }^{3}$ L. R. Pratt, Mol. Phys. 40, 347 (1980).

${ }^{4}$ J. S. Høye and G. Stell, J. Chem. Phys. 73, 461 (1980).

${ }^{5}$ F. Lado, Phys. Rev. E 55, 426 (1997).

${ }^{6}$ C. G. Gray and K. E. Gubbins, Theory of Molecular Fluids (Clarendon, Oxford, 1984), Vol. 1.

${ }^{7}$ J. P. Hansen and I. R. McDonald, Theory of Simple Liquids (Academic, London, 1986).

${ }^{8}$ P. M. Morse and H. Feshbach, Methods of Theoretical Physics (McGrawHill, New York, 1953), p. 1662 ff.

${ }^{9}$ See, for example, G. Arfken, Mathematical Methods for Physicists (Academic, Orlando, 1985).

${ }^{10}$ Handbook of Mathematical Functions, edited by M. Abramowitz and I. A. Stegun (Dover, New York, 1965), Chap. 22. 